\title{
Application of infrared image restoration to improve the accuracy of surface temperature measurements
}

\author{
by E.R. Meinders, T.H. van der Meer and K. Hanjalić \\ Delft University of Technology, Faculty of Applied Physics, P.O. Box 5046, 2600 GA, Delft, The Netherlands
}

\begin{abstract}
Image degradation is regarded as the major limiting factor for accurate evaluation of surface temperatures with infrared thermography, especially if a high spatial resolution in combination with a large depth-of-field is required. The paper discusses the application of an infrared image restoration technique to enhance the accuracy of surface temperature measurements of small three-dimensional objects. The restoration technique applied is based on a Wiener filter which uses the two-dimensional Optical Transfer Function (OTF), characterising the infrared camera. The OTF was determined from the camera response to a perfect thermal step-edge. Infrared thermographs, acquired from small cubical elements subject to well established boundary conditions, are processed with both the restoration filter and in situ calibration to arrive at surface temperatures. Surface temperatures, obtained independently with liquid crystal thermography, are in excellent agreement with the restored results, showing both the necessity for image restoration and improvement of accuracy.
\end{abstract}

\section{Introduction}

Infrared surface temperature measurements are often accompanied by spatial image degradation. Particularly in cases with three-dimensional objects when a high spatial resolution and depth-of-field are required, image degradation becomes the limiting factor in accurate surface temperature analysis. A lack of desired accuracy in the temperature measurement is directly projected in the evaluation of heat transfer phenomena, especially in laboratory applications. A sufficient accuracy can only be achieved by employing a restoration technique, based on the two-dimensional optical transfer function (OTF). In the past decade, there has been a substantial research focussed on different techniques to characterise infrared systems using the Modulation Transfer Function (MTF), which is the magnitude of the Optical Transfer Function (OTF) (see for example [1-5]). Although [4] presented restored images, an evidence of the improvement of the temperature accuracy after image restoration was not provided.

This paper focusses on a restoration technique in a particular application to the measurement of the surface temperatures of small cubical elements. These cubes are used to simulate the local convective heat transfer in complex flow situations, as occurring in the interior of an electronic cabinet [6]. The image restoration technique uses a Wiener filter which was constructed from the OTF, characterising the low wavelength infrared camera used in the present analysis. Infrared thermographs, acquired from the cubical elements with well established boundary conditions, are processed with both the restoration filter and an in situ calibration to arrive at surface temperatures. in order to validate the accuracy improvement of the infrared measurements, the restored infrared results are compared with surface temperature measurements obtained independently from liquid crystal thermography under similar experimental conditions [6]. 


\section{Experimental setup and measurement technique}

The experimental setup consists of an array of cubical elements, $15 \mathrm{~mm}$ in size, mounted on an insulated base-plate, which defines one of the vertical walls of a windtunnel. Typical free stream velocities are between 0.5 and $6 \mathrm{~m} / \mathrm{s}$. The elements consist of an internal copper core covered by a thin epoxy layer. The elements are powered by means of a heat source, embedded in the copper core. The local convective heat transfer, as a result of the complex flow pattern around the elements, as well as the three-dimensional geometry result in high spatial temperature gradients at the surface of the elements. Surface temperatures of all five faces, exposed to airflow, are required to calculate the distribution of the convective heat flux over the cube surface. Since the cubes are located in a closed wind-tunnel, the four side faces of the cube, perpendicular to the mounting base, are scanned under an angle of 45 degrees. A schematical sketch of this set-up is given in figure 1 . The angled projections require a depth-of-field of the infrared camera of at least $\pm 5 \mathrm{~mm}$. The cubes are painted with a high emissivity paint for enhanced radiation. Experiments showed a uniform emissivity of 0.95 for scan-angles up to approximately $55-60$ degrees.

\section{Infrared thermography}

\subsection{Infrared system}

A low-wavelength infrared imaging system (Varioscan, Jenoptik) with response between 2 and 5.5 micron was used. The camera contains a two-dimensional scanning mechanism to create images of 200 lines, each consisting of 300 pixels. The dynamic range that we used ensured a thermal resolution of at least $0.2^{\circ} \mathrm{C}$ for the investigated situations. An object distance of 0.19 $\mathrm{m}$ was selected during all experiments, corresponding to a sample density of approximately 5 pixels $/ \mathrm{mm}$.

\subsection{In situ calibration}

The unambiguous relation between the actual surface temperature of the element and the camera read-out (spatial pixel-values) was obtained with an in situ calibration, incorporating all kind of environmental influences, e.g. reflections from hot neighbouring components, transmissivity of the used wind-tunnel foil, etc. The measurement elements are replaced by an identical copper element with similar dimensions $(15 \times 15 \times 15 \mathrm{~mm})$ and surface emissivity. Variation of the copper temperature with incremental temperature steps yields calibration-curves, describing the relation between emitted intensity and surface temperature for each particular location at the cube-surface. Figure 2 shows a series of typical calibration curves for 15 surface cells located at the centreline of the top face of the cube. These calibration curves are based upon restored infrared images of the calibration object. The discrepancies in intensity between different locations correspond to approximately $0.5-1^{\circ} \mathrm{C}$, illustrating the necessity of this in situ calibration. A detailed discussion on this in situ calibration technique is given in [6]. The infrared technique with this in situ calibration involves a spatial accuracy within $0.5 \mathrm{~mm}$.

\subsection{Spatial image degradation}

Spatial image degradation, a consequence of optical diffraction and aberration blur, is the main source of temperature inaccuracy, especially when small objects are studied for which a high spatial resolution and large depth-of-field are required. The blurred image can be recovered with a restoration filter, characteristic for the used infrared system. Considering the problem in the Fourier domain, the restored image $\hat{\imath}\left(f_{x}, f_{y}\right)$ becomes [7]:

$$
\hat{I}\left(f_{x}, f_{y}\right)=W\left(f_{x}, f_{y}\right) M\left(f_{x}, f_{y}\right)
$$


where $M\left(f_{x}, f_{y}\right)$ is the blurred image, $W\left(f_{x}, f_{y}\right)$ is the restoration filter and $f_{x}$ and $f_{y}$ are the spatial frequencies. The Wiener restoration filter is based on the optical transfer function (OTF) and reads [7]:

$$
W\left(f_{x}, f_{y}\right)=\frac{H^{*}\left(f_{x}, f_{y}\right)}{\left|H\left(f_{x}, f_{y}\right)\right|^{2}+\left(P_{\text {noise }} / P_{\text {image }}\right)}
$$

where $H\left(f_{x}, f_{y}\right)$ and $H^{*}\left(f_{x}, f_{y}\right)$ are the optical transfer function and its complex conjugate, respectively. $P_{\text {noise }}$ and $P_{\text {image }}$ are the power spectral densities of the noise and image, respectively. The ratio $P_{\text {image }} / P_{\text {noise }}$ is the signal-to-noise-ratio of the image.

\subsection{Two-dimensional OTF}

Although an infrared system is characterised by its OTF, it is often easier to acquire Line Spread Functions (LSF), from which the OTF can be derived. The camera response to a perfect thermal step-edge was used to determine the LSFs. The thermal step-edge was made with a sharp step in surface emissivity of a heated copper plate. One half of the plate was covered with a very thin coating $(0.01 \mathrm{~mm})$ with emissivity $\epsilon_{1}$ and the other half with a thin coating with $\epsilon_{2}$. The high thermal conductivity of copper and the very small layer-thickness of the coating ensured that the temperature of the entire surface of the test-plate was uniform (within $0.05^{\circ} \mathrm{C}$ ). The difference in surface emissivity of the test-plate causes a significant difference in emitted radiation. The modulus of the Fourier transform of a typical LSF, better known as the modulation transfer function (MTF), is given in figure 3. The object distance is $0.19 \mathrm{~m}$. This MTF is peaked in the low spatial frequency region. The pronounced plateau is attributed to the camera properties. The maximum spatial frequency encountered in presented results was $2900.21 / \mathrm{m}$, corresponding to a pixel size of $0.3448 \mathrm{~mm}$. A two-dimensional OTF was derived from these typical one-dimensional camera responses. The corresponding magnitude is shown in figure 4.

An extensive discussion on the measurement techniques and accuracies involved to acquire the LSFs and final OTF is given in [8]. Measurements of the OTF for small out-of-focus situations verified the required depth-of-field of at least $\pm 5 \mathrm{~mm}$. A test-image was restored with these small defocussed OTFs, yielding temperature discrepancies smaller than $0.1{ }^{\circ} \mathrm{C}$. Wavelength dependency of the restoration filter was eliminated by measurements of OTFs for different temperature levels of the thermal step-edge. The test-image, restored with Wiener filters based on these different OTFs, showed temperature discrepancies within $0.1^{\circ} \mathrm{C}$. Therefore, a unique OTF was acquired which is applicable for the temperature range of interest $\left(40-65^{\circ} \mathrm{C}\right)$. An extensive discussion is given in [8].

\section{Temperature measurements}

Liquid crystal thermography is used as a second independent measurement technique to validate the temperature results acquired from the infrared image restoration technique. Thermochromic liquid crystals are substances with the properties of both a liquid and a crystalline state, giving the relation between colour and temperature. The properties, difficulties and applications concerning these thermochromic liquid crystals for surface temperature measurements are discussed in detail in [9].

A mixture of 5 liquid crystals is used in this particular study, resulting in 5 temperature events. An appropriate calibration method, as described in [6], is used to determine the temperatures at the crossings between green and red, i.e. $47.5,55.8,60.5,65.2$ and $72.8^{\circ} \mathrm{C}$. Although only surface temperatures at distinct locations are acquired, this approach of considering only the crossings provides an objective determination of the surface temperature. The accuracy of these temperatures, evaluated with the standard single sample uncertainty analysis described by [10], is within $0.3-0.4^{\circ} \mathrm{C}$. The spatial accuracy of the temperature measurements performed with liquid crystals is within $0.5 \mathrm{~mm}$. 
Well controlled boundary conditions (power supply, air velocity, air temperature, etc.) are maintained during the surface temperature measurements with the infrared and the liquid crystal technique in order to allow for a comparison between both measurement techniques. Surface temperatures are presented for three different convective cooling rates, i.e. velocities of 0.8 , 2.1 and $5.1 \mathrm{~m} / \mathrm{s}$, resulting in three different surface temperature maps. Figure 5 shows the comparison between liquid crystal measurements, denoted as $(\triangle)$, and infrared thermography measurements along trajectories $A B C D$ and $A B C D A$, which are perpendicular and parallel to the mounting base, respectively. Infrared measurements which are processed with the in situ calibration but not restored with the Wiener restoration technique are presented as ( $\square$ ). Despite of using the in situ calibration, the discrepancies between both techniques are unacceptably large. However, the surface temperatures obtained after infrared image restoration, denoted as $(\diamond)$, are in good agreement with the liquid crystal measurements, illustrating the necessity of image restoration. Liquid crystal point measurements and restored infrared thermography measurements of the temperature along trajectories $A B C D$ and $A B C D A$ are collected in figure 6 for the three mentioned cooling rates. The small discontinuities at the corners are attributed to the somewhat larger inaccuracy in surface temperature as is caused by the mapping of the different faces. The small discrepancies between both techniques, provided the employment of the in situ calibration and image restoration technique, confirm the estimated averaged accuracy of 0.3-0.4 ${ }^{\circ} \mathrm{C}$.

\section{Conclusion}

This paper is focussed on the image restoration technique to improve the accuracy of surface temperature measurements with an infrared system. The findings can be summarised as follows: - The applicability of a thermal imaging system to measure large surface temperature gradients on small objects with a high absolute accuracy of $0.3-0.4^{\circ} \mathrm{C}$ and a large spatial resolution is shown.

- The two-dimensional optical transfer function, acquired from measurements of a perfect thermal step-edge, was used to construct a Wiener restoration filter.

- Infrared images, acquired from cubical elements with large spatial temperature gradients, were processed with the restoration filter as well with an in situ calibration to arrive at accurate surface temperatures.

- The surface temperatures were also measured with liquid crystal thermography during well controlled environmental conditions. The good agreement between both independent techniques after application of the restoration technique, proves that the applied image restoration for this kind of applications is acceptably accurate and reliable.

\section{Acknowledgements}

The authors would like to express their gratitude to Geert van Kempen and Lucas van Vliet for their collaboration in the development of the restoration technique.

\section{REFERENCES}

[1] TZANNES (A.P.) and MOONEY (J.M.).- Measurement of the modulation transfer function of infrared cameras. Optical Engineering, Vol. 34 no. 6, 1995, p. 1808-1817.

[2] DANIELS (A.), BOREMAN (G.D.) \& DUCHARME (A.D.).- Random transparency targets for modulation transfer function measurements in the visible and infrared regions. Optical Engineering, Vol. 34 no. 3,1995 , p. 860-868.

[3] WILLIAMS (T.L.).- The MTF of thermal imaging cameras - its relevance and measurements. Proceedings, Quantitative Infrared thermography, QIRT 92, Paris, France, 1992, p. 51-56.

[4] Bougeard (D.), VERMEULEN (J.P.) and BAUDOIN (B.).- Mesure du champ de temperature sur une ailette d'echangeur par thermographie infrarouge. Revue Generale de Thermique, Vol. 34 no. 400-401, 1995, p. 325-334. 
[5] CARLOMAGNO (G.M.).- Quantitative infrared thermography. Proceedings, 2nd European Thermal-Sciences and 14th UIT National Heat Transfer Conference, Rome, Italy, Vol 1, 1996, p. 93-101.

[6] MEINDERS (E.R.), VAN DER MEER (T.H.), HANJALIĆ (K.) and LASANCE (C.J.M.).- Application of infrared thermography to the evaluation of local convective heat transfer on arrays of cubical protrusions. Int. J. Heat and Fluid Flow, Vol 18 No 1, 1997, p. 152-159.

[7] JAIN (A.K.).- Fundamentals of digital image processing. Prentice-Hall International, Inc, Englewood Cliffs, NJ 07632, 1989.

[8] MEINDERS (E.R.), VAN KEMPEN (G.M.P.), VAN VLIET (L.J.) \& VAN DER MEER (T.H.).Measurement and application of an infrared image restoration filter to improve the accuracy of surface temperature measurements of cubes. 1997, submitted for publication consideration.

[9] MOFFAT (R.J.).- Experimental Heat Transfer. Proceedings, 9th International Heat Transfer Conference, Jerusalem, Israel, Vol. 1, 1990, p. 187-205.

[10] MOFFAT (R.J.).- Describing the uncertainties in experimental results. Experimental Thermal and Fluid Science, Vol. 1 no. 1, 1988, p. 3-17.

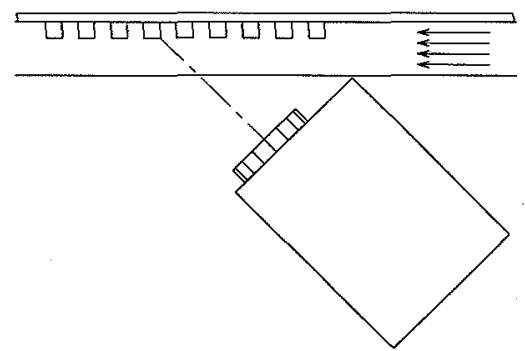

Figure 1: Experimental set-up of the infrared camera for scanning the east-top projection

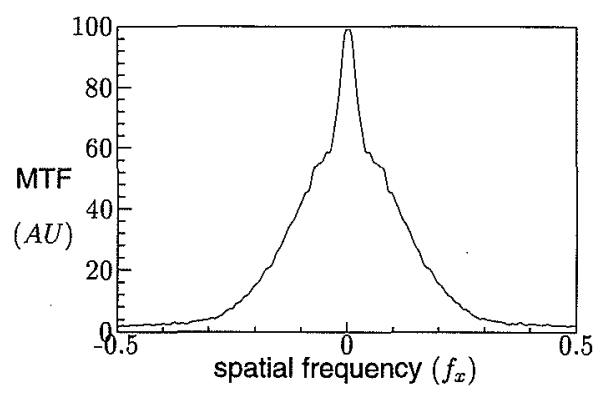

Figure 3: One-dimensional MTF for the horizontal scan-direction.

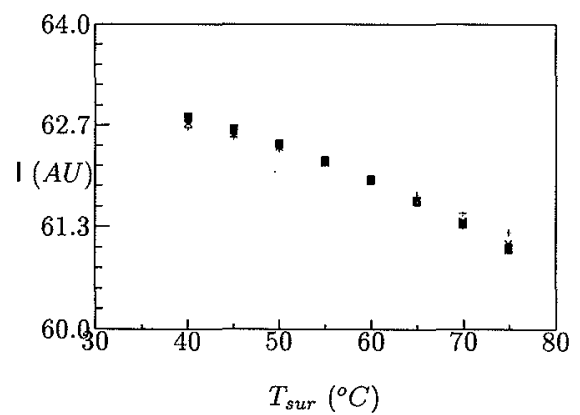

Figure 2: In situ calibration curves for surface cells at the centreline of the top face: intensities versus surface temperatures.

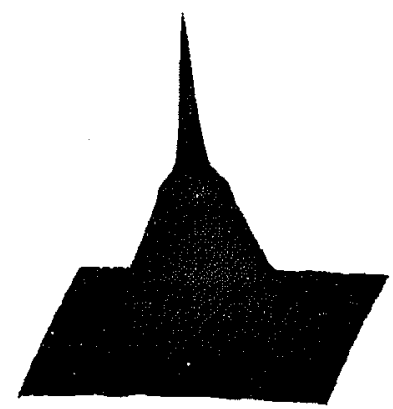

Figure 4: Two-dimensional optical transfer function $H\left(f_{x}, f_{y}\right)$. 

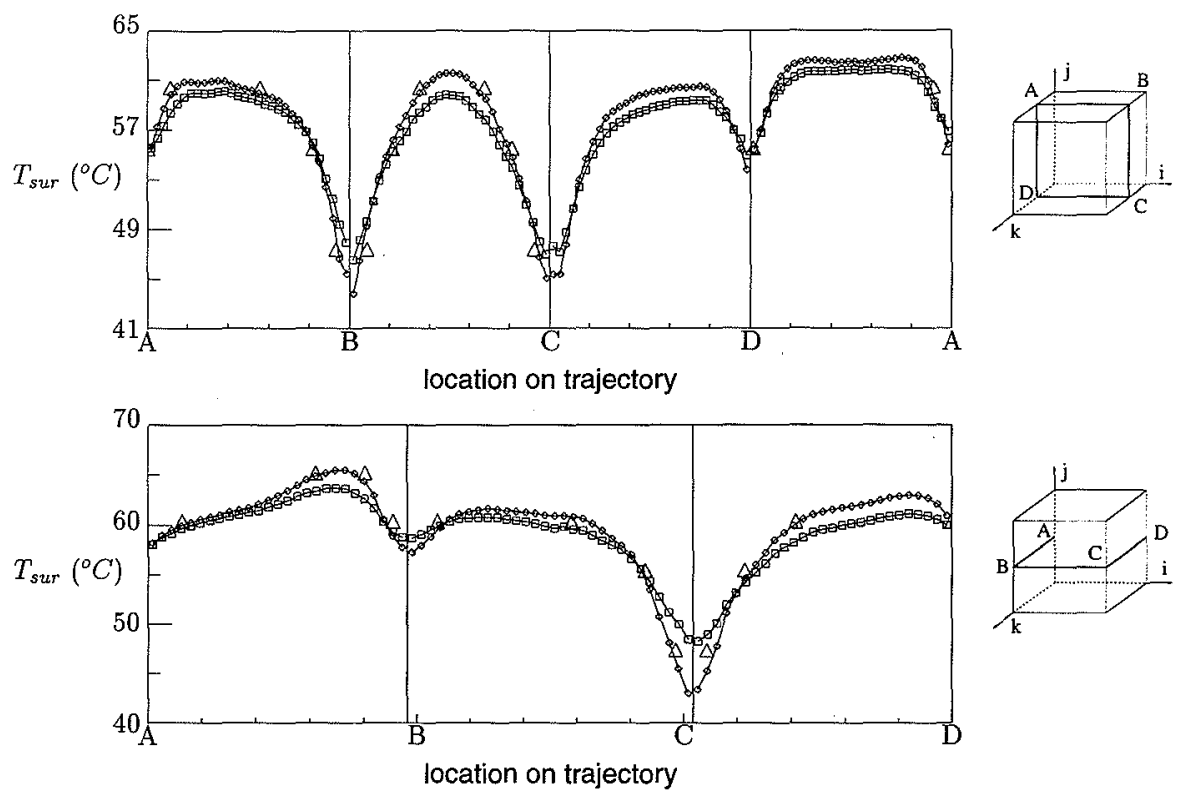

Figure 5: Comparison of surface temperature measurements between liquid crystal thermography $(\triangle)$ and infrared results before $(\square)$ and after $(\diamond)$ application of the restoration technique for two different trajectories.
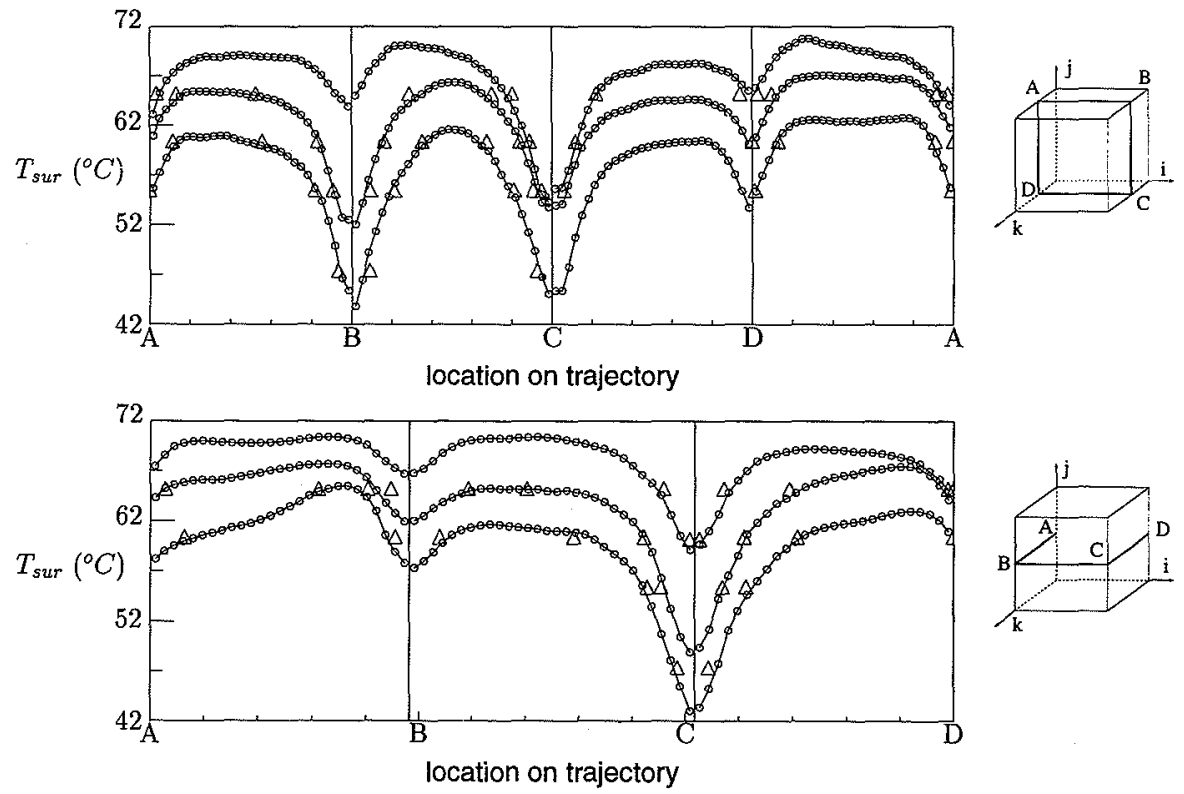

Figure 6: Comparison between restored infrared surface temperature measurements (o) and liquid crystal thermography $(\triangle)$ for two different trajectories for respectively $0.8,2.1$ and $5.1 \mathrm{~m} / \mathrm{s}$. 